\title{
Some identities involving the partial sum of $q$-binomial coefficients
}

\author{
Bing $\mathrm{He}$ \\ Department of Mathematics, Shanghai Key Laboratory of PMMP \\ East China Normal University \\ 500 Dongchuan Road, Shanghai 200241, People's Republic of China \\ yuhe001@f oxmail.com
}

Submitted: Feb 21, 2014; Accepted: Jul 21, 2014; Published: Jul 25, 2014

Mathematics Subject Classifications: 05A10; 05A15

\begin{abstract}
We give some identities involving sums of powers of the partial sum of $q$-binomial coefficients, which are q-analogues of Hirschhorn's identities [Discrete Math. 159 (1996), 273-278] and Zhang's identity [Discrete Math. 196 (1999), 291-298].
\end{abstract}

Keywords: binomial coefficients, $q$-binomial coefficients, $q$-binomial theorem

\section{Introduction}

In [2], Calkin proved the following curious identity:

$$
\sum_{k=0}^{n}\left(\sum_{j=0}^{k}\left(\begin{array}{l}
n \\
j
\end{array}\right)\right)^{3}=n \cdot 2^{3 n-1}+2^{3 n}-3 n\left(\begin{array}{c}
2 n \\
n
\end{array}\right) 2^{n-2}
$$

Hirschhorn [5] established the following two identities on sums of powers of binomial partial sums:

$$
\sum_{k=0}^{n} \sum_{j=0}^{k}\left(\begin{array}{l}
n \\
j
\end{array}\right)=n \cdot 2^{n-1}+2^{n}
$$

and

$$
\sum_{k=0}^{n}\left(\sum_{j=0}^{k}\left(\begin{array}{l}
n \\
j
\end{array}\right)\right)^{2}=n \cdot 2^{2 n-1}+2^{2 n}-\frac{n}{2}\left(\begin{array}{c}
2 n \\
n
\end{array}\right) .
$$


In [7], Zhang proved the following alternating form of (2):

$$
\sum_{k=0}^{n}(-1)^{k}\left(\sum_{j=0}^{k}\left(\begin{array}{l}
n \\
j
\end{array}\right)\right)^{2}= \begin{cases}1, & \text { if } n=0, \\
2^{2 n-1}, & \text { if } n \text { is even and } n \neq 0 \\
-2^{2 n-1}-(-1)^{(n-1) / 2}\left(\begin{array}{c}
n-1 \\
(n-1) / 2
\end{array}\right), & \text { if } n \text { is odd. }\end{cases}
$$

Several generalizations are given in $[6,8,9]$. Later, Guo et al. [4] gave the following $q$-identities:

$$
\sum_{k=0}^{2 n}(-1)^{k}\left(\sum_{j=0}^{k}\left[\begin{array}{c}
2 n \\
j
\end{array}\right]_{q}\right)^{2}=\left(\sum_{k=0}^{2 n}\left[\begin{array}{c}
2 n \\
k
\end{array}\right]_{q}\right)\left(\sum_{k=0}^{n}\left[\begin{array}{c}
2 n \\
2 k
\end{array}\right]_{q}\right)
$$

and

$$
\begin{aligned}
\sum_{k=0}^{2 n+1}(-1)^{k}\left(\sum_{j=0}^{k}\left[\begin{array}{c}
2 n+1 \\
j
\end{array}\right]_{q}\right)^{2}= & -\left(\sum_{k=0}^{n}\left[\begin{array}{c}
2 n+1 \\
2 k
\end{array}\right]_{q}\right)\left(\sum_{k=0}^{2 n+1}\left[\begin{array}{c}
2 n+1 \\
k
\end{array}\right]_{q}\right) \\
& -\sum_{k=0}^{n}(-1)^{k}\left[\begin{array}{c}
2 n+1 \\
k
\end{array}\right]_{q}^{2}-2 \sum_{0 \leqslant i<j \leqslant n}(-1)^{i}\left[\begin{array}{c}
2 n+1 \\
i
\end{array}\right]_{q}\left[\begin{array}{c}
2 n+1 \\
j
\end{array}\right]_{q}
\end{aligned}
$$

Here and in what follows, $\left[\begin{array}{l}n \\ k\end{array}\right]_{q}$ is the $q$-binomial coefficient defined by

$$
\left[\begin{array}{l}
n \\
k
\end{array}\right]_{q}= \begin{cases}\frac{(q ; q)_{n}}{(q ; q)_{k}(q ; q)_{n-k}}, & \text { if } 0 \leqslant k \leqslant n \\
0, & \text { otherwise }\end{cases}
$$

where $(z ; q)_{n}=(1-z)(1-z q) \cdots\left(1-z q^{n-1}\right)$ is the $q$-shifted factorial for $n \geqslant 0$.

The purpose of this paper is to study $q$-analogues of (1)-(2) and establish a new $q$-version of (3). Our main results may be stated as follows.

Theorem 1. For any positive integer $n$ and any non-zero integer $m$, we have

$$
\sum_{k=0}^{n} \sum_{j=0}^{k}\left[\begin{array}{l}
n \\
j
\end{array}\right]_{q} q^{m k+\left(\begin{array}{l}
j \\
2
\end{array}\right)}=\frac{\left(-q^{m}, q\right)_{n}-q^{m(n+1)}(-1, q)_{n}}{1-q^{m}},
$$

and

$$
\begin{aligned}
& \sum_{k=0}^{n} q^{-k}\left(\sum_{i=0}^{k}\left[\begin{array}{c}
n \\
i
\end{array}\right]_{q} q^{\left(\begin{array}{c}
i \\
2
\end{array}\right)}\right)\left(\sum_{j=0}^{k}\left[\begin{array}{l}
n \\
j
\end{array}\right]_{q} q^{\left(\begin{array}{l}
j \\
2
\end{array}\right)+2(1-n) j}\right) \\
& \quad=\frac{\left(\left(-q^{-1} ; q\right)_{n}-q^{-(n+1)}(-1 ; q)_{n}\right)\left(-q^{2(1-n)} ; q\right)_{n}}{1-q^{-1}}-\sum_{i=0}^{n-1} \frac{1-q^{n-i}}{1-q}\left[\begin{array}{c}
2 n \\
i
\end{array}\right]_{q} q^{\left(\begin{array}{l}
i \\
2
\end{array}\right)-\frac{3 n^{2}}{2}+\frac{n}{2}+1} .
\end{aligned}
$$


Theorem 2. For any non-negative integer n, we have

$$
\begin{gathered}
\sum_{k=0}^{2 n+1}(-1)^{k}\left(\sum_{i=0}^{k}\left[\begin{array}{c}
2 n+1 \\
i
\end{array}\right]_{q} q^{\left(\begin{array}{c}
i \\
2
\end{array}\right)}\right)\left(\sum_{j=0}^{k}\left[\begin{array}{c}
2 n+1 \\
j
\end{array}\right]_{q} q^{\left(\begin{array}{c}
2 n-j+1 \\
2
\end{array}\right)}\right) \\
=-q^{2 n^{2}+n}\left(-q^{-2 n} ; q\right)_{4 n+1}-\sum_{i=0}^{n}(-1)^{i}\left[\begin{array}{c}
2 n+1 \\
i
\end{array}\right]_{q^{2}} q^{2\left(\begin{array}{c}
i \\
2
\end{array}\right)},
\end{gathered}
$$

and

$$
\sum_{k=0}^{2 n+2}(-1)^{k}\left(\sum_{i=0}^{k}\left[\begin{array}{c}
2 n+2 \\
i
\end{array}\right]_{q} q^{\left(\begin{array}{c}
i \\
2
\end{array}\right)}\right)\left(\sum_{i=0}^{k}\left[\begin{array}{c}
2 n+2 \\
i
\end{array}\right]_{q} q^{\left(\begin{array}{c}
2 n+2-i \\
2
\end{array}\right)}\right)=q^{2 n^{2}+3 n+1}\left(-q^{-1-2 n} ; q\right)_{4 n+3} .
$$

Letting $q \rightarrow 1$ and using L'Hôpital's rule and some familiar identities, we easily find that the identities (4)-(5) and (6)-(7) are $q$-analogues of (1)-(2) and (3) respectively.

In Sections 2 and 3, we will give proofs of Theorems 1.1 and 1.2 respectively by using the $q$-binomial theorem and generating functions.

\section{Proof of Theorem 1.1}

To give our proof of Theorem 1.1, we need to establish a result, which is a $q$-analogue of Chang and Shan's identity (see [3]).

Lemma 3. For any positive integer n, we have

$$
\sum_{k=0}^{n-1} q^{-k}\left(\sum_{i=0}^{k}\left[\begin{array}{c}
n \\
i
\end{array}\right]_{q} q^{\left(\begin{array}{c}
i \\
2
\end{array}\right)}\right)\left(\sum_{j=k+1}^{n}\left[\begin{array}{c}
n \\
j
\end{array}\right]_{q} q^{\left(\begin{array}{c}
j \\
2
\end{array}\right)+2(1-n) j}\right)=\sum_{i=0}^{n-1} \frac{1-q^{n-i}}{1-q}\left[\begin{array}{c}
2 n \\
i
\end{array}\right]_{q} q^{\left(\begin{array}{c}
i \\
2
\end{array}\right)-\frac{3 n^{2}}{2}+\frac{n}{2}+1}
$$

Proof. According to the $q$-binomial theorem (see [1]), we have for all complex numbers $z$ and $q$ with $|z|<1$ and $|q|<1$, there holds

$$
(z, q)_{n}=\sum_{k=0}^{n}(-1)^{k}\left[\begin{array}{l}
n \\
k
\end{array}\right]_{q} q^{\left(\begin{array}{l}
k \\
2
\end{array}\right) z^{k}}
$$

and

$$
\frac{1}{(z, q)_{n}}=\sum_{i \geqslant 0}\left[\begin{array}{c}
n+i-1 \\
i
\end{array}\right]_{q} z^{i}
$$

It follows that

$$
\begin{gathered}
(-z ; q)_{n} \frac{1}{1-z}=\left(\sum_{i=0}^{n}\left[\begin{array}{c}
n \\
i
\end{array}\right]_{q} q^{\left(\begin{array}{c}
i \\
2
\end{array}\right) z^{i}}\right)\left(\sum_{i=0}^{\infty} z^{i}\right), \\
\left(-z q^{n} ; q\right)_{n} \frac{1}{1-z q}=\left(\sum_{i=0}^{n}\left[\begin{array}{c}
n \\
i
\end{array}\right]_{q} q^{\left(\begin{array}{c}
i \\
2
\end{array}\right)+n i} z^{i}\right)\left(\sum_{i=0}^{\infty} q^{i} z^{i}\right),
\end{gathered}
$$


and

$$
(-z ; q)_{2 n} \frac{1}{(z ; q)_{2}}=\left(\sum_{i=0}^{2 n}\left[\begin{array}{c}
2 n \\
i
\end{array}\right]_{q} q^{\left(\begin{array}{c}
i \\
2
\end{array}\right)} z^{i}\right)\left(\sum_{i=0}^{\infty}\left[\begin{array}{c}
1+i \\
i
\end{array}\right]_{q} z^{i}\right) .
$$

Therefore, for any non-negetive integer $k$ with $k \leqslant n-1$, the coefficient of $z^{k}$ in $(-z ; q)_{n} \frac{1}{1-z}$ is

$$
\sum_{i=0}^{k}\left[\begin{array}{l}
n \\
i
\end{array}\right]_{q} q^{\left(\begin{array}{c}
i \\
2
\end{array}\right)}
$$

the coefficient of $z^{n-k-1}$ in $\left(-z q^{n} ; q\right)_{n} \frac{1}{(1-z q}$ is

$$
\sum_{i=k+1}^{n}\left[\begin{array}{l}
n \\
i
\end{array}\right]_{q} q^{\left(\begin{array}{c}
n-i \\
2
\end{array}\right)+n(n-i)+i-k-1}
$$

and the coefficient of $z^{n-1}$ in $(-z ; q)_{2 n} \frac{1}{(z ; q)_{2}}$ is

$$
\sum_{i=0}^{n-1}\left[\begin{array}{c}
2 n \\
i
\end{array}\right]_{q} \frac{1-q^{n-i}}{1-q} q^{\left(\begin{array}{c}
i \\
2
\end{array}\right)}
$$

Using the fact

$$
(-z ; q)_{n} \frac{1}{1-z} \cdot\left(-z q^{n} ; q\right)_{n} \frac{1}{1-z q}=(-z ; q)_{2 n} \frac{1}{(z ; q)_{2}},
$$

equating the coefficients of $z^{n-1}$ and after some simplifications, we obtain Lemma 2.1.

Proof of Theorem 1.1. We first prove (4).

$$
\begin{aligned}
\sum_{k=0}^{n} \sum_{j=0}^{k}\left[\begin{array}{l}
n \\
j
\end{array}\right]_{q} q^{m k+\left(\begin{array}{l}
j \\
2
\end{array}\right)} & =\sum_{j=0}^{n}\left[\begin{array}{l}
n \\
j
\end{array}\right]_{q} q^{\left(\begin{array}{l}
j \\
2
\end{array}\right) \sum_{k=j}^{n} q^{m k}} \\
& =\frac{\sum_{j=0}^{n}\left[\begin{array}{l}
n \\
j
\end{array}\right]_{q} q^{\left(\begin{array}{c}
j \\
2
\end{array}\right)+m j}-q^{m(n+1)} \sum_{j=0}^{n}\left[\begin{array}{c}
n \\
j
\end{array}\right]_{q} q^{\left(\begin{array}{l}
j \\
2
\end{array}\right)}}{1-q^{m}} \\
& =\frac{\left(-q^{m}, q\right)_{n}-q^{m(n+1)}(-1, q)_{n}}{1-q^{m}}
\end{aligned}
$$

where in the last step, we have used (8).

We next show (5). By (8), we have

$$
\sum_{j=0}^{n}\left[\begin{array}{l}
n \\
j
\end{array}\right]_{q} q^{\left(\begin{array}{l}
j \\
2
\end{array}\right)+2(1-n) j}=\left(-q^{2(1-n)} ; q\right)_{n}
$$

and taking $m=-1$ in (4), we obtain

$$
\sum_{k=0}^{n} q^{-k} \sum_{j=0}^{k}\left[\begin{array}{l}
n \\
j
\end{array}\right]_{q} q^{\left(\begin{array}{l}
j \\
2
\end{array}\right)}=\frac{\left(-q^{-1}, q\right)_{n}-q^{-(n+1)}(-1, q)_{n}}{1-q^{-1}} .
$$


Hence, by Lemma 2.1, we get

$$
\begin{aligned}
& \sum_{k=0}^{n} q^{-k}\left(\sum_{i=0}^{k}\left[\begin{array}{l}
n \\
i
\end{array}\right]_{q} q^{\left(\begin{array}{c}
i \\
2
\end{array}\right)}\right)\left(\sum_{j=0}^{k}\left[\begin{array}{l}
n \\
j
\end{array}\right]_{q} q^{\left(\begin{array}{c}
j \\
2
\end{array}\right)+2(1-n) j}\right) \\
& =\sum_{k=0}^{n} q^{-k}\left(\sum_{i=0}^{k}\left[\begin{array}{l}
n \\
i
\end{array}\right]_{q} q^{\left(\begin{array}{c}
i \\
2
\end{array}\right)}\right)\left(\left(-q^{2(1-n)} ; q\right)_{n}-\sum_{j=k+1}^{n}\left[\begin{array}{c}
n \\
j
\end{array}\right]_{q} q^{\left(\begin{array}{c}
j \\
2
\end{array}\right)+2(1-n) j}\right) \\
& =\left(-q^{2(1-n)} ; q\right)_{n} \sum_{k=0}^{n} q^{-k}\left(\sum_{i=0}^{k}\left[\begin{array}{l}
n \\
i
\end{array}\right]_{q} q^{\left(\begin{array}{c}
i \\
2
\end{array}\right)}\right) \\
& -\sum_{k=0}^{n-1} q^{-k}\left(\sum_{i=0}^{k}\left[\begin{array}{l}
n \\
i
\end{array}\right]_{q} q^{\left(\begin{array}{l}
i \\
2
\end{array}\right)}\right)\left(\sum_{j=k+1}^{n}\left[\begin{array}{l}
n \\
j
\end{array}\right]_{q} q^{\left(\begin{array}{l}
j \\
2
\end{array}\right)+2(1-n) j}\right) \\
& =\frac{\left(\left(-q^{-1} ; q\right)_{n}-q^{-(n+1)}(-1 ; q)_{n}\right)\left(-q^{2(1-n)}, q\right)_{n}}{1-q^{-1}}-\sum_{i=0}^{n-1} \frac{1-q^{n-i}}{1-q}\left[\begin{array}{c}
2 n \\
i
\end{array}\right]_{q} q^{\left(\begin{array}{c}
i \\
2
\end{array}\right)-\frac{3 n^{2}}{2}+\frac{n}{2}+1} \text {. }
\end{aligned}
$$

\section{Proof of Theorem 1.2}

In order to prove the Theorem 1.2, we need the following result, which gives a $q$-analogue of alternating sums of Chang and Shan's identity.

Lemma 4. For any non-negative integer $n$, we have

$$
\sum_{k=0}^{2 n}(-1)^{k}\left(\sum_{i=0}^{k}\left[\begin{array}{c}
2 n+1 \\
i
\end{array}\right]_{q} q^{\left(\begin{array}{c}
i \\
2
\end{array}\right)}\right)\left(\sum_{j=k+1}^{2 n+1}\left[\begin{array}{c}
2 n+1 \\
j
\end{array}\right]_{q} q^{\left(\begin{array}{c}
2 n-j+1 \\
2
\end{array}\right)}\right)=\sum_{i=0}^{n}(-1)^{i}\left[\begin{array}{c}
2 n+1 \\
i
\end{array}\right]_{q^{2}} q^{2\left(\begin{array}{c}
i \\
2
\end{array}\right)} .
$$

Proof. By (8), we find that

$$
\begin{gathered}
(z ; q)_{n} \frac{1}{1+z}=\left(\sum_{i=0}^{n}\left[\begin{array}{c}
n \\
i
\end{array}\right]_{q} q^{\left(\begin{array}{c}
i \\
2
\end{array}\right)}(-z)^{i}\right)\left(\sum_{i=0}^{\infty}(-z)^{i}\right), \\
(-z ; q)_{n} \frac{1}{1-z}=\left(\sum_{i=0}^{n}\left[\begin{array}{c}
n \\
i
\end{array}\right]_{q} q^{\left(\begin{array}{c}
i \\
2
\end{array}\right) z^{i}}\right)\left(\sum_{i=0}^{\infty} z^{i}\right),
\end{gathered}
$$

and

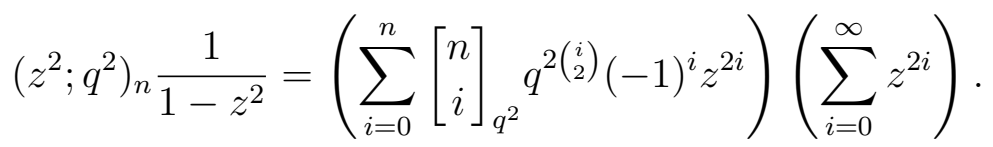

Therefore, for any non-negetive integer $k$ with $k \leqslant n-1$, the coefficient of $z^{k}$ in $(z ; q)_{n} \frac{1}{1+z}$ is

$$
(-1)^{k} \sum_{i=0}^{k}\left[\begin{array}{l}
n \\
i
\end{array}\right]_{q} q^{\left(\begin{array}{c}
i \\
2
\end{array}\right)}
$$


the coefficient of $z^{n-k-1}$ in $(-z ; q)_{n} \frac{1}{1-z}$ is

$$
\sum_{i=k+1}^{n}\left[\begin{array}{l}
n \\
i
\end{array}\right]_{q} q^{\left(\begin{array}{c}
n-i \\
2
\end{array}\right)}
$$

and the coefficient of $z^{n-1}$ in $\left(z^{2} ; q^{2}\right)_{n} \frac{1}{1-z^{2}}$ is

$$
\sum_{i=0}^{(n-1) / 2}(-1)^{i}\left[\begin{array}{l}
n \\
i
\end{array}\right]_{q^{2}} q^{2\left(\begin{array}{l}
i \\
2
\end{array}\right)}[2 \mid(n-1)],
$$

where $[2 \mid n]$ is defined by

$$
[2 \mid n]= \begin{cases}1, & \text { if } 2 \mid n \\ 0, & \text { otherwise }\end{cases}
$$

Using the fact

$$
(-z ; q)_{n} \frac{1}{1-z} \cdot(z ; q)_{n} \frac{1}{1+z}=\left(z^{2} ; q^{2}\right)_{n} \frac{1}{1-z^{2}}
$$

equating the coefficients of $z^{n-1}$ and after some simplifications, we obtain Lemma 3.1.

Proof of Theorem 1.2. We first prove (6). By (8), we have

$$
\begin{aligned}
\sum_{k=0}^{n}(-1)^{k} \sum_{j=0}^{k}\left[\begin{array}{l}
n \\
j
\end{array}\right]_{q} q^{\left(\begin{array}{c}
j \\
2
\end{array}\right)} & =\sum_{j=0}^{n}\left[\begin{array}{l}
n \\
j
\end{array}\right]_{q} q^{\left(\begin{array}{c}
j \\
2
\end{array}\right)} \sum_{k=j}^{n}(-1)^{k} \\
& =\frac{1}{2} \sum_{j=0}^{n}\left[\begin{array}{l}
n \\
j
\end{array}\right]_{q} q^{\left(\begin{array}{c}
j \\
2
\end{array}\right)}\left((-1)^{j}-(-1)^{n+1}\right) \\
& =\frac{(-1)^{n}}{2}(-1, q)_{n}
\end{aligned}
$$

and

$$
\sum_{j=0}^{2 n+1}\left[\begin{array}{c}
2 n+1 \\
j
\end{array}\right]_{q} q^{\left(\begin{array}{c}
2 n-j+1 \\
2
\end{array}\right)}=q^{2 n^{2}+n}\left(-q^{-2 n} ; q\right)_{2 n+1} .
$$

Replacing $n$ by $2 n+1$ in (9), we obtain

$$
\sum_{k=0}^{2 n+1}(-1)^{k}\left(\sum_{i=0}^{k}\left[\begin{array}{c}
2 n+1 \\
i
\end{array}\right]_{q} q^{\left(\begin{array}{c}
i \\
2
\end{array}\right)}\right)=-(-q ; q)_{2 n} .
$$


Hence, by Lemma 3.1, we get

$$
\begin{aligned}
& \sum_{k=0}^{2 n+1}(-1)^{k}\left(\sum_{i=0}^{k}\left[\begin{array}{c}
2 n+1 \\
i
\end{array}\right]_{q} q^{\left(\begin{array}{c}
i \\
2
\end{array}\right)}\right)\left(\sum_{j=0}^{k}\left[\begin{array}{c}
2 n+1 \\
j
\end{array}\right]_{q} q^{\left(\begin{array}{c}
2 n-j+1 \\
2
\end{array}\right)}\right) \\
& \left.=\sum_{k=0}^{2 n+1}(-1)^{k}\left(\sum_{i=0}^{k}\left[\begin{array}{c}
2 n+1 \\
i
\end{array}\right]_{q} q^{\left(\begin{array}{c}
i \\
2
\end{array}\right)}\right)\left(q^{2 n^{2}+n}\left(-q^{-2 n} ; q\right)_{2 n+1}-\sum_{j=k+1}^{2 n+1}\left[\begin{array}{c}
2 n+1 \\
j
\end{array}\right]_{q} q^{(2 n-j+1}\right)\right) \\
& =-q^{2 n^{2}+n}\left(-q^{-2 n} ; q\right)_{4 n+1}-\sum_{k=0}^{2 n}(-1)^{k}\left(\sum_{i=0}^{k}\left[\begin{array}{c}
2 n+1 \\
i
\end{array}\right]_{q} q^{\left(\begin{array}{c}
i \\
2
\end{array}\right)}\right)\left(\sum_{j=k+1}^{2 n+1}\left[\begin{array}{c}
2 n+1 \\
j
\end{array}\right]_{q}^{\left.q_{2}^{(2 n-j+1}\right)}\right) \\
& =-q^{2 n^{2}+n}\left(-q^{-2 n} ; q\right)_{4 n+1}-\sum_{i=0}^{n}(-1)^{i}\left[\begin{array}{c}
2 n+1 \\
i
\end{array}\right]_{q^{2}} q^{2\left(\begin{array}{c}
i \\
2
\end{array}\right)} .
\end{aligned}
$$

We next show (7). By (8), we have

$$
\left.\sum_{j=0}^{2 n}\left[\begin{array}{c}
2 n \\
j
\end{array}\right]_{q} q^{(2 n-j}\right)=q^{2 n^{2}-n}\left(-q^{1-2 n} ; q\right)_{2 n}
$$

and replacing $n$ by $2 n$ in (9), we obtain

$$
\sum_{k=0}^{2 n}(-1)^{k}\left(\sum_{i=0}^{k}\left[\begin{array}{c}
2 n \\
i
\end{array}\right]_{q} q^{\left(\begin{array}{c}
i \\
2
\end{array}\right)}\right)=(-q ; q)_{2 n-1}
$$

Hence, by the fact

$$
\sum_{k=0}^{2 n-1}(-1)^{k}\left(\sum_{i=0}^{k}\left[\begin{array}{c}
2 n \\
i
\end{array}\right]_{q} q^{\left(\begin{array}{c}
i \\
2
\end{array}\right)}\right)\left(\sum_{i=k+1}^{2 n}\left[\begin{array}{c}
2 n \\
i
\end{array}\right]_{q} q^{\left(\begin{array}{c}
2 n-i \\
2
\end{array}\right)}\right)=0
$$

which follows easily from the substitution $k \rightarrow 2 n-1-k$, we have

$$
\begin{aligned}
& \sum_{k=0}^{2 n}(-1)^{k}\left(\sum_{i=0}^{k}\left[\begin{array}{c}
2 n \\
i
\end{array}\right]_{q} q^{\left(\begin{array}{c}
i \\
2
\end{array}\right)}\right)\left(\sum_{i=0}^{k}\left[\begin{array}{c}
2 n \\
i
\end{array}\right]_{q} q^{\left(\begin{array}{c}
2 n-i \\
2
\end{array}\right)}\right) \\
& =\sum_{k=0}^{2 n}(-1)^{k}\left(\sum_{i=0}^{k}\left[\begin{array}{c}
2 n \\
i
\end{array}\right]_{q} q^{\left(\begin{array}{c}
i \\
2
\end{array}\right)}\right)\left(q^{2 n^{2}-n}\left(-q^{1-2 n} ; q\right)_{2 n}-\sum_{i=k+1}^{2 n}\left[\begin{array}{c}
2 n \\
i
\end{array}\right]_{q} q^{\left(\begin{array}{c}
2 n-i \\
2
\end{array}\right)}\right) \\
& =q^{2 n^{2}-n}\left(-q^{1-2 n} ; q\right)_{4 n-1} .
\end{aligned}
$$

\section{Acknowledgement}

I would like to thank the referee for his/her helpful comments. 


\section{References}

[1] G.E. Andrews, The Theory of Partitions, Cambridge University Press, Cambridge, 1998.

[2] N.J. Calkin, A curious binomial identity, Discrete Math. 131 (1994), 335-337.

[3] G.-Z. Chang, Z. Shan, Problems 83-3: A binomial summation, SIAM Review, 1983, 25(1): 97.

[4] V.J.W. Guo, Y.-J. Lin, Y. Liu, C. Zhang, A q-analogue of Zhang's binomial coefficient identities, Discrete Math. 309 (2009), 5913-5919.

[5] M. Hirschhorn, Calkin's binomial identity, Discrete Math. 159 (1996), 273-278.

[6] J, Wang, Z. Zhang, On extensions of Calkin's binomial identities, Discrete Math. 274 (2004), 331-342.

[7] Z. Zhang, A kind of binomial identity, Discrete Math. 196(1999), 291-298.

[8] Z. Zhang, J. Wang, Generalization of a combinatorial identity, Util. Math. 71 (2006), $217-224$.

[9] Z. Zhang, X. Wang, A generalization of Calkin's identity, Discrete Math. 308 (2008), 3992-3997. 